\title{
The effect of varying temperature and $\mathrm{O}_{2}$ flow rate in ex situ annealed tin-doped indium for fabrication of commercial grade indium tin oxide
}

\author{
M. R. Kiyani • Y. S. Jalili
}

Received: 11 May 2014 / Accepted: 10 June 2014/Published online: 28 August 2014

(C) The Author(s) 2014. This article is published with open access at Springerlink.com

\begin{abstract}
In this work, tin-doped indium was deposited on a glass substrate via the electron beam evaporation technique. Then, the as-grown thin films were baked in the presence of oxygen at different $\mathrm{O}_{2}$ flow rates and temperatures inside a furnace to obtain transparent conducting oxide thin film structures. The electrical and optical properties of the layers were investigated, the thickness of all samples was kept at $500 \mathrm{~nm}$ and the rate of deposition was set at $0.1 \mathrm{~nm} \mathrm{~min}{ }^{-1}$. The best optical and electrical properties

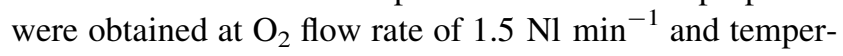
atures of $500{ }^{\circ} \mathrm{C}$ where above $90 \%$ optical transparency and $\leq 4 \times 10^{-3} \mathrm{ohm} \mathrm{cm}^{-1}$ electrical resistivity were achieved.
\end{abstract}

Keywords ITO - Transparent conductors · Annealing · Transparency $\cdot$ Resistivity $\cdot$ Sputtering

\section{Introduction}

Transparent conducting oxides (TCO) are used mainly in optoelectronic applications such as flat panel displays, thin film transistors, electroluminescent devices, solar cells, gas sensors and light emitting diodes [1-5]. The value of electrical conductivity depends on various factors, one of which is the concentration of the dopant incorporated. However, the increase in conductivity usually lowers the transparency level. Hence, it is necessary to find a balance between high optical transparency and high electrical conductivity.

M. R. Kiyani · Y. S. Jalili

Department of Physics, Science and Research Branch,

IAU. P. O. Box: 14665-678, Tehran, Iran

Y. S. Jalili ( $\square)$

SheykhBahaee Experimental Research Center, Science \& Research Branch, IAU P. O. Box: 14665-678, Tehran, Iran e-mail: seyedjalili@srbiau.ac.ir
Indium tin oxide (ITO) is the most popular among the TCO materials, used particularly for transparent electrodes in LCD, PLED and OLED displays, solar cells and also as antistatic coatings. ITO is usually grown by RF-sputtering reactively in the presence of oxygen. The strong dependence of ITO electro-optical properties on oxygen pressure and stoichiometry ratio during the growth process [4] adds extra difficulties to this technique during the growth process. The basic properties of ITO oxides are primarily low electrical resistivity (of the order of $10^{-3} \mathrm{ohm} \mathrm{cm}^{-1}$ at room temperature) and high optical transparency (about $90 \%$ in the visible range or the optical spectrum) and high infrared reflectance [2].

In the current study, indium-tin thin films are grown by electron beam evaporation technique, with $\mathrm{O}_{2}$ being a reactive gas and hence advantageous to be excluded at the growth stage, and implemented under $\mathrm{O}_{2}$-free conditions. The film samples were then baked inside a furnace under exposure to $\mathrm{O}_{2}$ flow to obtain ITO thin films. This process was carried out under varying furnace temperatures and $\mathrm{O}_{2}$ flow rates, and their effects on the electro-optical properties of the ITO thin film samples were investigated.

\section{Experimental}

In (Aldrich $99.99 \%$ ) and Sn (Aldrich 99.99\%) powder were purchased from Sigma, and were mixed to obtain indium-tin with a ratio of 9:1. After having been fully grinded and mixed in a mortar, it was then cast into pellets of $1 \mathrm{~cm}$ in diameter using a pellet press under force of $2,500 \mathrm{~N}$. To enhance cohesion in the pellets, a drop of acetone was added to the powder before being pressed.

Prior to deposition, the lamellae substrates were first rinsed with deionized water and then they were sonicated 
Table 1 Bake setting according to oxygen flow rates, baking temperature and time duration of as-grown tin-doped indium thin films

\begin{tabular}{lllll}
\hline $\begin{array}{l}\text { Sample } \\
\text { no. }\end{array}$ & $\begin{array}{l}\text { Temperature } \\
\left({ }^{\circ} \mathrm{C}\right)\end{array}$ & $\begin{array}{l}\text { Oxygen flow } \\
\left(1 \mathrm{~min}^{-1}\right)\end{array}$ & $\begin{array}{l}\text { Time } \\
(\mathrm{min})\end{array}$ & $\begin{array}{l}\text { Oxygen } \\
\text { pressure (bar) }\end{array}$ \\
\hline $\mathbf{1}$ & 500 & 0.5 & 30 & 1.5 \\
$\mathbf{2}$ & 500 & 1 & 30 & 1.5 \\
$\mathbf{3}$ & 500 & 1.5 & 30 & 1.5 \\
$\mathbf{4}$ & 400 & 0.5 & 30 & 1.5 \\
$\mathbf{5}$ & 400 & 1 & 30 & 1.5 \\
$\mathbf{6}$ & 400 & 1.5 & 30 & 1.5 \\
$\mathbf{7}$ & 300 & 0.5 & 30 & 1.5 \\
$\mathbf{8}$ & 300 & 1 & 30 & 1.5 \\
$\mathbf{9}$ & 300 & 1.5 & 30 & 1.5 \\
\hline
\end{tabular}

in propanol for $10 \mathrm{~min}$. Finally, they were washed with acetone and dried using pure $\mathrm{N}_{2}$ compressed gas. The resulting pellets and substrates were placed under electron beam evaporation gun (EDS-160). The distance between the indium-tin pellets target and substrate was set at $10 \mathrm{~cm}$. Pressure was set at $1.5 \times 10^{-5}$ tor and the atmosphere was rendered oxygen-free. Voltage was kept constant at $6,000 \mathrm{~V}$ and the current was gradually increased to $16 \mathrm{~A}$, where evaporation was initiated. The deposition was carried at a rate of $0.1 \mathrm{~nm} \mathrm{~min}{ }^{-1}$. According to the thickness monitor, once the thickness of the as-grown layers reached $500 \mathrm{~nm}$, deposition was terminated.

The as-grown tin-doped indium samples were then baked at varying temperatures and times inside a furnace under exposure to oxygen with varying flow rates to obtain ITO thin films. The baking settings for each substrate sample are given in Table 1; the rate of heating/cooling was kept at $4{ }^{\circ} \mathrm{C} \min ^{-1}$ throughout the baking process.

All the analyses were carried out in 1 day to prevent any aging effects. SEM analysis was carried out with the aid of Hitachi model S-4160 and AFM analysis was implemented using a Park Scientific Instrument model auto probe CP, the optical transmittance spectra of films in the UV-Vis-NIR region were recorded by a spectrophotometer (Cary 500 Scan).

\section{Results and discussion}

The crystallinity and crystal orientation of ITO thin films were measured using X-ray diffraction (STADI MP) with a
Fig. 1 X-ray diffraction pattern of ITO thin films by ex situ baking of as-grown tindoped indium thin films onto glass substrate at $\mathbf{a}$ at $500^{\circ}, \mathbf{b}$ at $400^{\circ}$ and $\mathbf{c}$ at $300^{\circ}$. The corresponding sample number is indicated on each spectra, see Table 1 for details
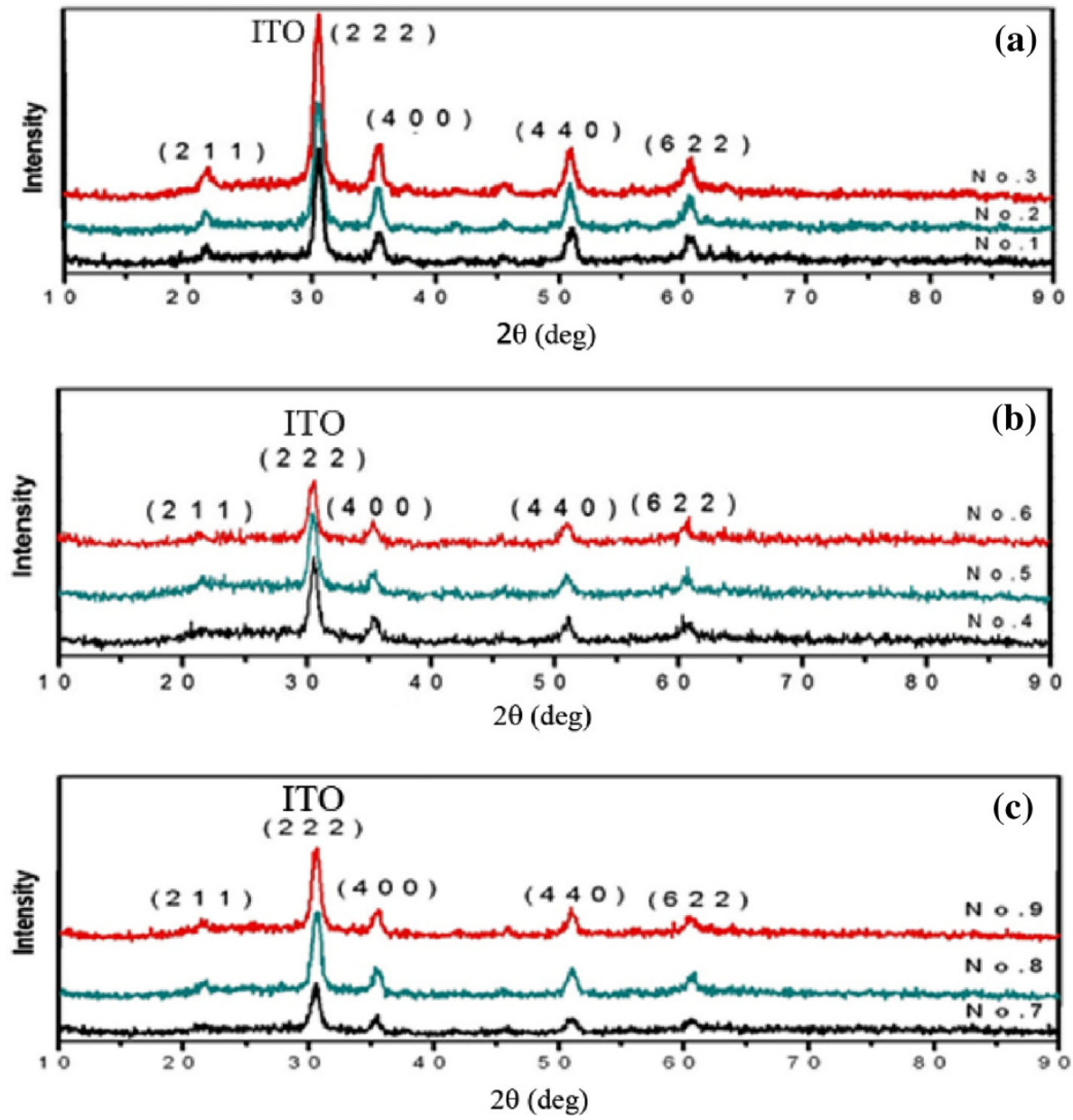
$\mathrm{CuKa}_{1}$ source $(\lambda=1.54)$. XRD spectra are shown in Fig. 1.

After baking under exposure to oxygen, five distinct peaks (211), (222), (400), (440) and (622) appeared for all of the ITO thin film samples in comparison to the as-grown tin-doped indium thin films which demonstrated amorphous structures as expected. This indicates crystallization within the thin film samples during the baking process in the presence of oxygen. The peaks at $2 \theta=30^{\circ}, 35^{\circ}, 51^{\circ}$ and $60^{\circ}$ indicate the existence of ITO. For some layers, raising the flow rate of oxygen results in the appearance of indium oxide peak at $2 \theta=21^{\circ}$. The fact that all spectra demonstrate a predominant peak at (222) plane indicates that the prepared films had a tendency to crystallize along a favorable orientation.

The UV-Vis-NIR spectra measurement is illustrated in Fig. 2. From the spectra, for the baked sample under $\mathrm{O}_{2}$ at $400{ }^{\circ} \mathrm{C}$ (samples no. 4-6), visible range transparency of more than $90 \%$ was achieved.

The results indicate that the transmittance of the films was significantly influenced by increasing the furnace temperature and raising the flow rate of oxygen. In general, the transmittance is highly dependent on the scattering effect. It is assumed that the increase in temperature accelerated the occurrence degree of crystallization, bringing the system to the fibrous microstructure more rapidly. Accordingly, the microstructure with the fibrous pattern had a higher value of transmittance due to the reduction of light scattering sites in the thin film structure.

Using the relation below, the band gap of the thin film ITO layers can be obtained and the results are illustrated in Fig. 3.

$\alpha=\frac{A}{h v}\left(h v-E_{\mathrm{g}}\right)^{n}$

In this equation, $\alpha$ is the absorption coefficient, $A$ is a constant, $h v$ is the incident photon energy, $E_{\mathrm{g}}$ the band gap and $n$ is the number dependent on the type of excitation. We have set $n=0.5[10,11]$.

In Fig. 3, the band gap of the samples (numbers 1-9) is plotted and compared. As illustrated, with the increase in the flow rate of oxygen at different temperatures, the band gap decreases. This is because as the flow rate of oxygen increases, ITO thin films are better and more efficiently crystallized and as the thin film structures become more crystalline, it is more prone to have a relatively smaller band gap. Also it is worth noting that, the layer baked at $500{ }^{\circ} \mathrm{C}$ and flow rate of $1.5 \mathrm{Nl} \mathrm{min}{ }^{-1}$ demonstrates higher crystallinity (see Fig. 1) and hence will be expected to have a relatively smaller band gap and increased conductivity.

Conductivity measurement results indicate that resistivity of commercial grade magnitude of the order of $10^{-3}$ $\mathrm{ohm} \mathrm{cm}^{-1}$ was achieved. Considering the omission of
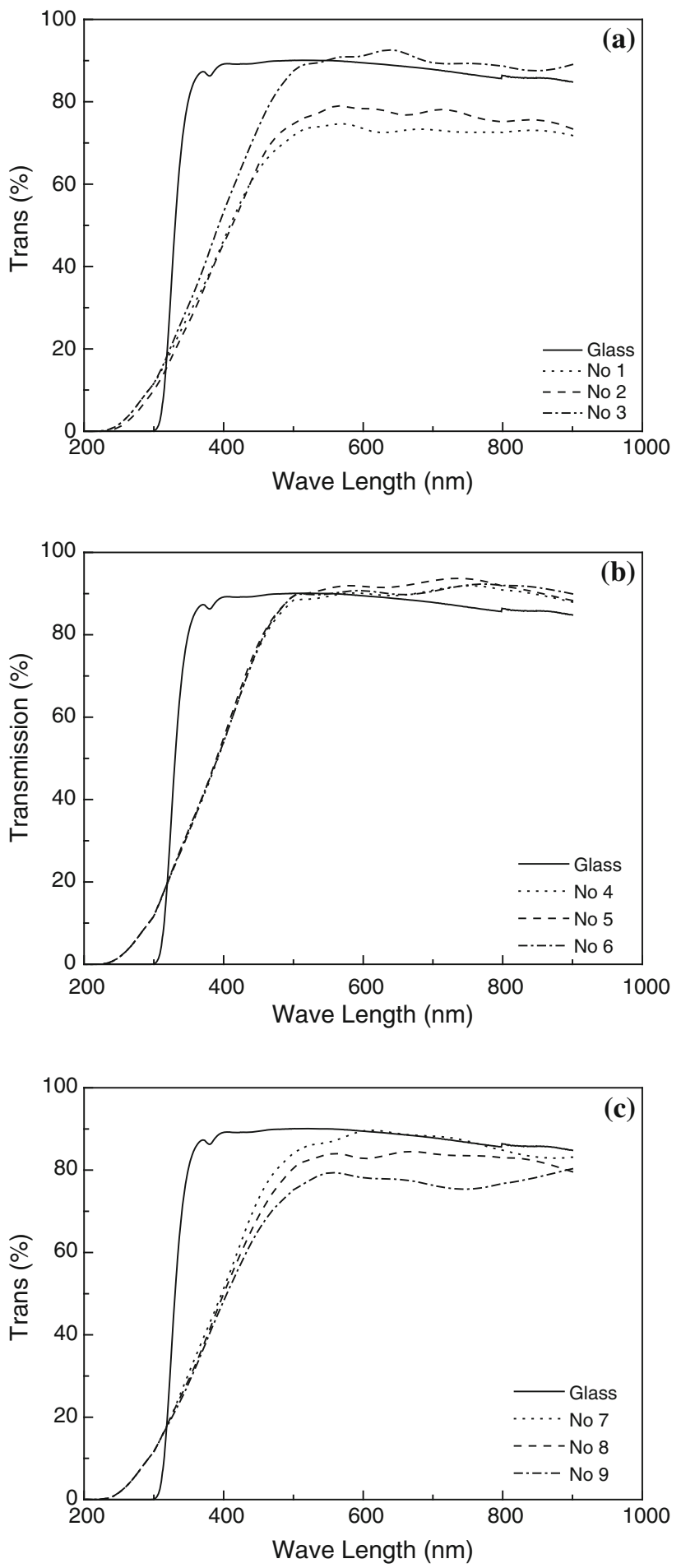

Fig. 2 Transmission spectra of the ITO thin film samples baked under exposure of $\mathrm{O}_{2}$ of varying flow rates at temperatures a $500^{\circ}$, b $400^{\circ}$ and c $300^{\circ}$. Note that Glass spectra are also included for reference

oxygen during the indium-tin growth process and its subsequent introduction into the ex situ thermal treatment stage, the $90 \%$ increase in transparency (Fig. 4) and the 


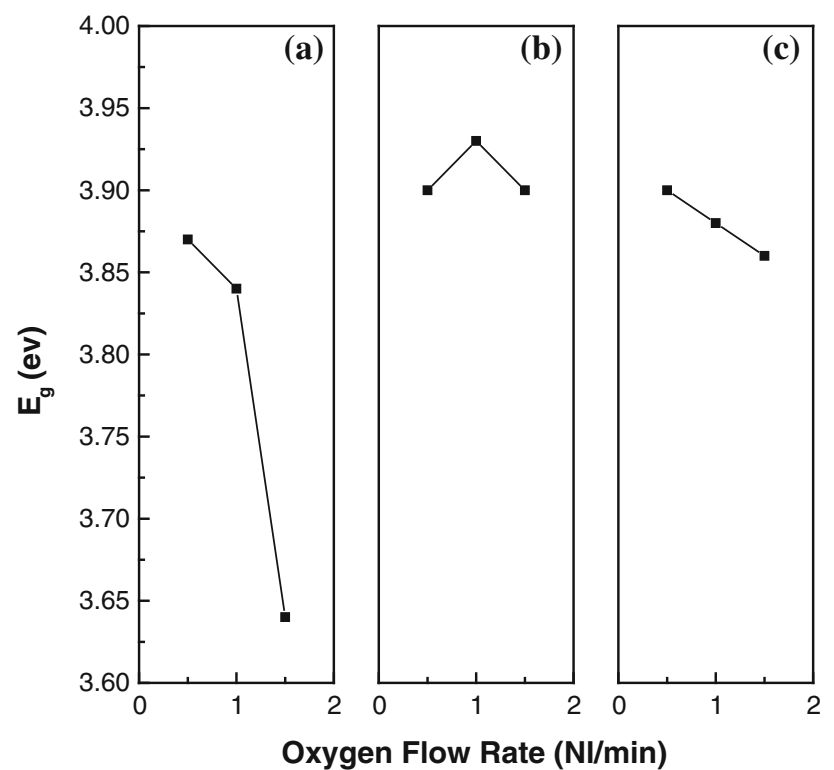

Fig. 3 The band gap of ITO samples baked at temperatures a $300{ }^{\circ} \mathrm{C}$, b $400{ }^{\circ} \mathrm{C}$ and c $500{ }^{\circ} \mathrm{C}$ using oxygen flow rates of 0.5 ,

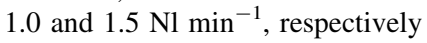

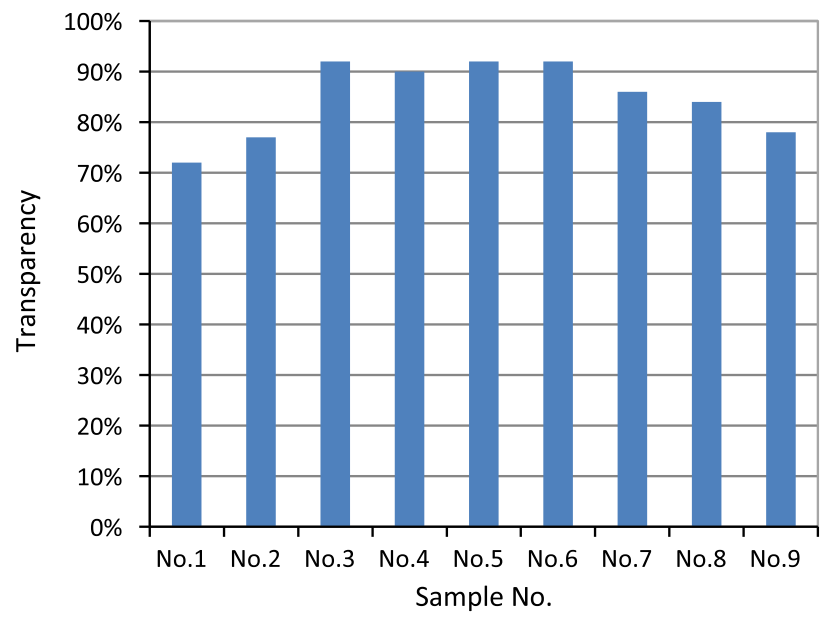

Fig. 4 Peak transmission of ITO thin films at $550 \mathrm{~nm}$ according to sample number, see Table 1 for details

decrease in resistance (Fig. 5) indicate that the foretold sample (no. 3) has a resistance of approximately commercial grade magnitude $\leq 4 \times 10^{-3} \mathrm{ohm} \mathrm{cm}^{-1}$. This was the best result obtained in this investigation.

In general, at $500{ }^{\circ} \mathrm{C}$, by increasing flow rate of oxygen, the resistivity of layers decreases systematically. It is clear that crystallization with higher flow rate of oxygen causes a reduction in sheet resistivity.

At $400{ }^{\circ} \mathrm{C}$, sheet resistivity of layers is approximately constant and higher than that at other temperatures. The transmittance of these layers is over $90 \%$. This indicates that the band gap increases and thereby sheet resistance also increases. Therefore, if we want to have layers with

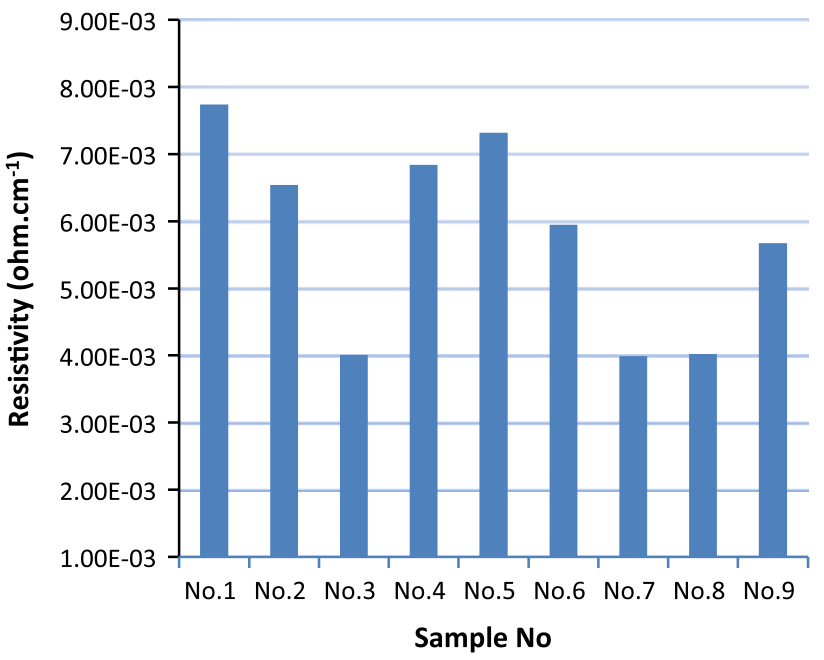

Fig. 5 Comparison of resistivity of ITO thin films according to sample number, see Table 1 for details

low resistivity, this temperature is not suitable for our purpose.

At $300{ }^{\circ} \mathrm{C}$, the initial resistivity of the ITO films is found to be approximately constant with oxygen flow rates

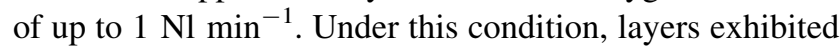
the lowest sheet resistance. The resistivity of ITO reached a

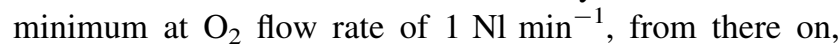
increasing the flow rate of oxygen resulted in a gradual increase in resistivity which is due to the formation of nonconducting O-rich clusters inside the film. Excess oxygen forms non-conducting O-rich clusters in the films resulting in crystal disorder, which act as trap sites and consequently increases the resistivity of the ITO thin films.

Overall, because of a relatively lower baking temperature at $300{ }^{\circ} \mathrm{C}$, as the $\mathrm{O}_{2}$ flow rate increases, non-conducting O-rich clusters in the film also increase; hence an increase in resistivity was observed $[7,8,9]$.

The high conductivity of the ITO films has been attributed to both substitutional tin and oxygen vacancies, created either during film growth or post-deposition annealing $[6,10,12]$. Therefore, at this temperature, the baking conditions seem not to be comparatively suitable for ITO formation from the as-grown tin-doped indium thin films.

The SEM images demonstrate that the unbaked asgrown tin-doped indium sample is void of crystallites. From Fig. 6b, d which correspond to sample with high resistance, crystallites are observed but the more obvious matter is the existence of trap sites between separate crystallites which bring about the observed increase in the electrical resistivity, this is also indicated by the AFM results.

As Fig. 6c corresponds to a sample with low resistivity and high transparency, it is well crystallized, as expected, 
Fig. 6 a Unbaked (as-grown) indium tin-doped thin film SEM image, b ITO thin film (sample no. 1) with low transparency and high resistance, $\mathbf{c}$ ITO thin film (sample no. 3) with high transparency and low resistivity, d ITO thin film (sample no. 5) with high transparency and high resistivity and e ITO thin film (sample no. 7) with low transparency and low resistivity
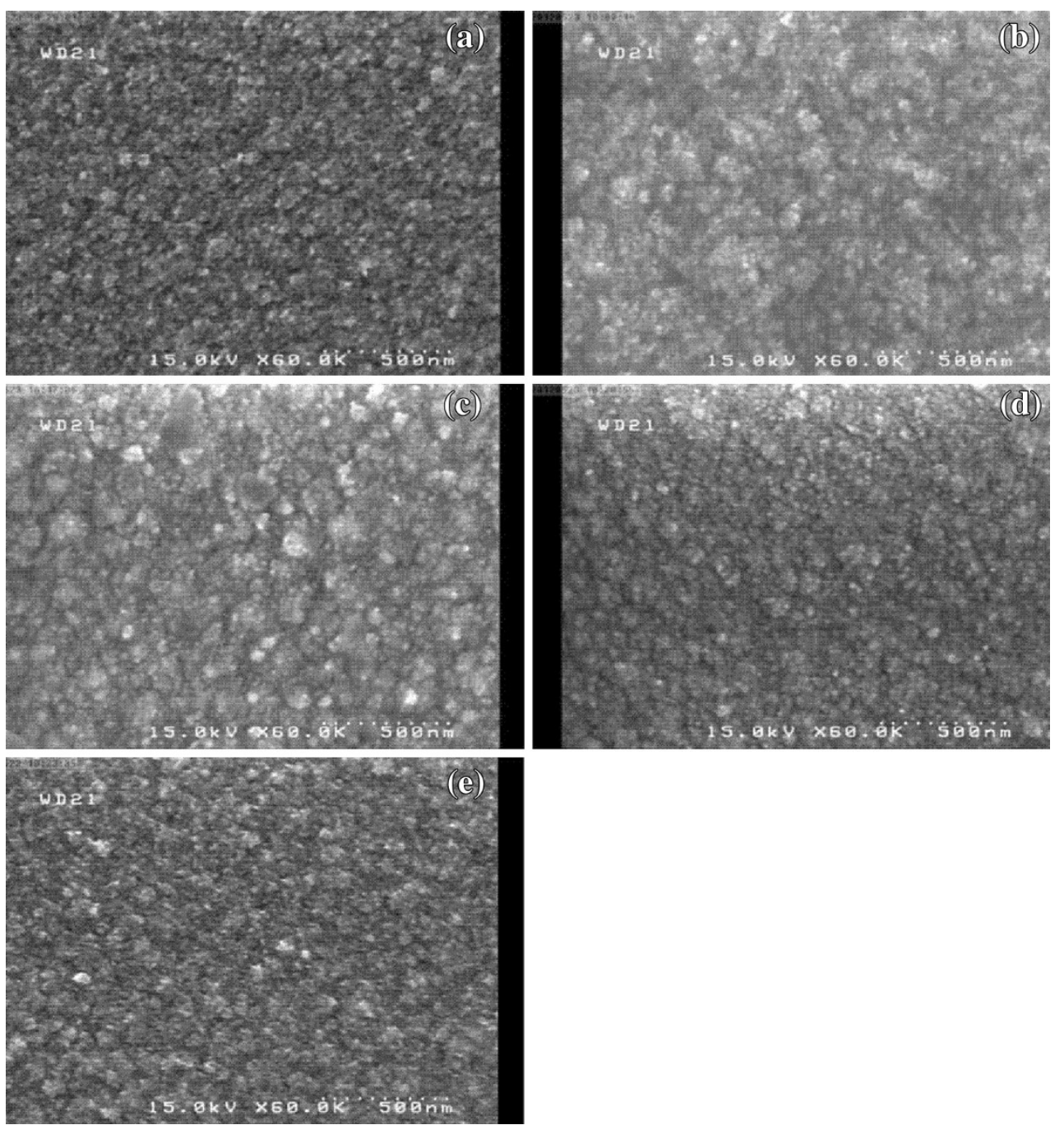

and one can easily observe the crystallites and the boundaries in between. Therefore, proper crystallization results in high transparency and low resistivity. As Fig. 6e illustrates, crystallites with low order result in low transparency and high resistivity.

AFM analysis was also performed for the three samples discussed above. The results are illustrated below in Fig. 7.

As indicated by the scanned images, the unbaked sample seems to be free of crystallites indicating a relatively much higher degree of roughness. In comparing the AFM image of sample no. 3 to that of sample no. 5, the particle size in sample no. 3 seems relatively larger than that observed in sample no. 5 and the degree of roughness observed is less than that of sample 5. Moreover, from the SEM image of sample no 5 , trap sites are more visible indicating a relative increase in the resistivity of the sample. Finally, it is worth noting that comparison of the roughnesses of samples 3 and 5 observed in AFM results may be an indication of the relative lower resistivity of sample no. 3 , considering the experimental observations given above.

The optoelectronic and structural properties of the ITO thin films are highly dependent on the deposition and baking conditions of the layers, such that any minuscule changes in these conditions result in notable changes in structural and conclusively in optoelectronic properties [5].

Regarding the lower resistivity of sample no. 3, if we consider the comparison of the indentations observed within the two samples 3 and 5 AFM images; sample no. 3 illustrates a lower degree of indentation to that observed in sample no. 5 this seems to ease the conduction process of the particles by omitting the necessity of tunneling.

\section{Conclusion}

Tin-doped indium oxide thin films were deposited using electron beam evaporation technique with an oxygen-free 

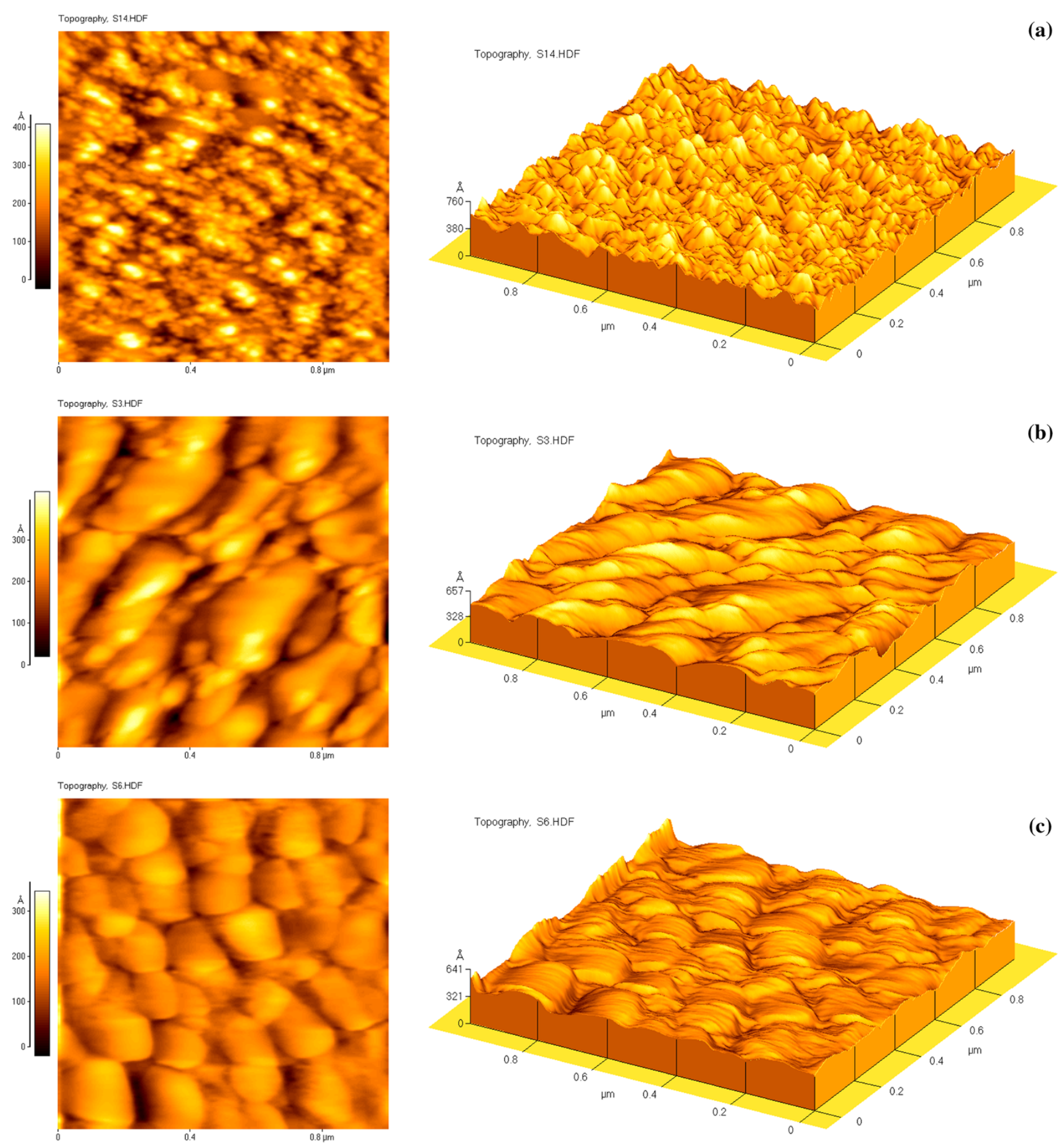

Fig. 7 a The as-grown (unbaked) tin-doped indium thin film AFM image, b ITO thin film baked at $500{ }^{\circ} \mathrm{C}$ and oxygen flow rate of $1.5 \mathrm{Nl} \mathrm{min}{ }^{-1}$ and $\mathbf{c}$ ITO thin film baked at $400{ }^{\circ} \mathrm{C}$ and oxygen flow rate of $1.0 \mathrm{Nl} \mathrm{min}{ }^{-1}$

environment. The resulting layers were baked in the presence of oxygen with different flow rates for $30 \mathrm{~min}$ at 300 , 400 and $500{ }^{\circ} \mathrm{C}$. XRD, UV-Vis spectrophotometry and four-point probe analysis show varying results. The experimental results indicate that the thin film baked at $500{ }^{\circ} \mathrm{C}$ with the oxygen flow rate of $1.5 \mathrm{Nl} \mathrm{min}{ }^{-1}$ is the layer with the optimal characteristics. After baking in an oxygen-containing environment, this layer exhibits low sheet resistance $\leq 4 \times 10^{-3} \mathrm{ohm} \mathrm{cm}^{-1}$ and a transmittance of $>90 \%$ within the visible range of the spectrum.

Open Access This article is distributed under the terms of the Creative Commons Attribution License which permits any use, distribution, and reproduction in any medium, provided the original author(s) and the source are credited. 


\section{References}

1. Hamberg, I., Granqvist, C.G.: Evaporated Sn-doped In2O3 films: basic optical properties and applications to energy-efficient windows. J. Appl. Phys. 60, R123 (1986)

2. You, Y.Z., kim, Y.S., Choi, D.H., jang, H.S.: Electrical and optical study of ITO films on glass and polymer substrates prepared by DC magnetron sputtering type negative metal ion beam deposition. Mater. Chem. Phys. 107, 444-448 (2008)

3. Ohta, H., Hosono, H.: Deposition of ITO using R.F magnetron sputtering. Thin Solid Films 413, 181-185 (2002)

4. Kim, H., Gilmore, C.M., Pique, A., Horwitz, J.S., Mattoussi, H., Murata, H., Kafafi, Z.H., Chrisey, D.B.: J. Appl. Phys. 86, 6451-6461 (1999)

5. Venkatachalam, S., Nanjo, H., Kawasaki, K., Hayashi, H., Ebina T., Mangalaraj, D.: Optoelectronic Properties of $\mathrm{ZnSe}$, ITO, $\mathrm{TiO}_{2}$ and $\mathrm{ZnO}$ thin films, optoelectronics-materials and techniques. In: Predeep, P. (ed.) InTech. doi:10.5772/18418. ISBN: 978-953307-276-0. http://www.intechopen.com/books/optoelectronicsmaterials-and-techniques/optoelectronic-properties-of-znse-itotio2-and-zno-thin-films

6. Cho, B.R., Park, I.Y.: Effect of post-annealing on the formation of ITO films. Korean Phys. Soc. 53, 19-22 (2008)

7. Pokaipisit, A., Horprathum, M., Limsuwan, P.: Influence of annealing temperature on the properties of ITO prepared by electron beam evaporation, Kasetsart. J. Nat. Sci. 42, 362-366 (2008)

8. Trejo-Cruz, C., Mendoza-Galvan, A., Lopez-Beltran, A.M.: Effect of air annealing on the optical, electrical, and structural properties of indium tin oxide film. Thin Solid Films 517, 4615-4620 (2009)

9. Zhou, Y., Kelly, P.J., Postill, A., Abu-zeid, O., Alnajjar, A.A.: The characteristics of aluminum-doped zinc oxide films prepared by pulsed magnetron sputtering from powder targets. Thin Solid Films 447, 33-39 (2009)

10. Aiempanakit, K., Rakkwamsuk, P., Grattana, D.: Characterization of indium tin oxide films after annealing in vacuum, Kasetsart. J. Nat. Sci. 42, 351 (2008)

11. Ho, C.Y., Tu, T.Y., Wang, C.C., Kang, Y: "Investigation of postannealing indium tin oxide for future electro-optical device application". In: Proceedings of the 10th WSEAS international conference on Telecommunications and informatics and microelectronics, nanoelectronics, optoelectronics, and World Scientific and Engineering Academy and Society (WSEAS) international conference on signal processing, pp. 154-158 (2011)

12. Fan, J.C.C., Goodenough, J.B.: X-ray photoemission spectroscopy studies of Sn-doped indium-oxide films. J. Appl. Phys. 48, 3524-3532 (1977) 